\title{
Prevalence of mutations and determinants of genotypic resistance to etravirine (TMC125) in a large ltalian resistance database (ARCA)* $\dagger$
}

\author{
P Di Vincenzo, ${ }^{1}$ S Rusconi, ${ }^{1}$ F Adorni, ${ }^{2}$ P Vitiello, ${ }^{1} \mathrm{~F}$ Maggiolo, ${ }^{3}$ D Francisci, ${ }^{4}$ A Di Biagio, ${ }^{5}$ L Monno, ${ }^{6}$ A Antinori, ${ }^{7}$ \\ E Boeri, ${ }^{8}$ G Punzi, ${ }^{6}$ C-F Perno, ${ }^{7}$ A Callegaro, ${ }^{3}$ B Bruzzone $^{5}$ and $\mathrm{M}$ Zazzi ${ }^{9}$ on behalf of the Antiretroviral Resistance Cohort \\ Analysis (ARCA) Collaborative Group \\ ${ }^{1}$ Sezione Malattie Infettive e Immunopatologia, Dipartimento di Scienze Cliniche 'Luigi Sacco', Universita' degli Studi, \\ Milan, Italy, ${ }^{2}$ ITB-CNR, Segrate (MI), Italy, ${ }^{3}$ Ospedali Riuniti, Bergamo, Italy, ${ }^{4}$ Perugia Hospital, Perugia, Italy, ${ }^{5}$ San Martino \\ Hospital, Genova, Italy, ${ }^{6}$ University of Bari, Bari, Italy, ${ }^{7}$ INMI 'Lazzaro Spallanzani' Hospital, Rome, Italy, ${ }^{8}$ HSR Scientific \\ Institute, Milan, Italy and ${ }^{9}$ University of Siena, Siena, Italy
}

\section{Objectives}

To evaluate whether etravirine (TMC125) might be effective in patients failing therapy with current nonnucleoside reverse transcriptase inhibitors (NNRTIs), we analysed the prevalence of TMC125 mutations and the possible determinants of genotypic resistance to this drug among sequences reported to a large database in Italy [Antiretroviral Resistance Cohort Analysis (ARCA)].

\section{Methods}

We analysed the prevalence of TMC125 resistance-associated mutations (RAMs) and the TMC125 weighted genotypic score (WGS) together with the determinants of genotypic resistance. A total of 5011 sequences from 2955 patients failing NNRTI therapy were evaluated.

\section{Results}

Among the sequences in ARCA, 68\% had at least one and 9.8\% at least three TMC125 RAMs, whereas 31\% had a WGS > 2. Frequent RAMs were Y181C, G190A, K101E and A98G, whereas V179F, Y181V and G190S appeared in < 5\% of sequences. Multivariate analysis revealed a higher risk of developing at least three TMC125 RAMs associated with both nevirapine and efavirenz exposure, whereas CD 4 counts $\geq 200$ cells/ $\mu \mathrm{L}$ retained their protective effect. An increased risk of WGS $>2$ was linked to higher HIV RNA values (maximum risk at $>5 \log _{10}$ copies $/ \mathrm{mL}$ ) and nevirapine exposure; CD4 counts $\geq 200$ cells/ $\mu \mathrm{L}$ were protective.

\section{Conclusions}

The prevalence of TMC125 resistance mutations in the ARCA cohort was 68\%. The DUET studies showed that at least three TMC125-associated mutations were required to impair the efficacy of the drug and Y181C/V, V179F and G190S had the greatest effect on response. The prevalence of these mutations among the patients examined in our study was low. However, WGS $>2$ was found for one-third of our sequences. Previous nevirapine exposure was associated with an increased risk of having WGS $>2$ (adjusted odds ratio 1.76).

Keywords: ARCA, drug resistance, genotype, HIV-1, TMC125

Accepted 23 November 2009

\footnotetext{
*This paper is dedicated to the memory of our friend Massimo Leporale. ${ }^{\dagger}$ This work was presented in part at the 6th European HIV Drug Resistance Workshop, Budapest, Hungary, 26-28 March 2008 (Abstract 59).

Correspondence: Dr Stefano Rusconi, Dipartimento di Scienze Cliniche 'Luigi Sacco', Sezione di Malattie Infettive e Immunopatologia, Universita' degli Studi di Milano, Via G.B. Grassi, 74, 20157 Milano, Italy. Tel: + 3902 39042668; fax: + 3902 50319758; e-mail: stefano.rusconi@unimi.it
}

\section{Introduction}

HIV-infected patients who experience triple-drug class virological failure may be at increased risk of clinical progression and death [1]. Therefore, newer agents with activity against drug-resistant HIV-1 are needed [2] 
Etravirine (TMC125) is a second-generation nonnucleoside reverse transcriptase inhibitor (NNRTI) with potent activity against wild-type and NNRTI-resistant HIV-1 and a high genetic barrier to the development of resistance [3,4]; its potent antiretroviral activity has been demonstrated in the phase III DUET studies [5,6].

Information on TMC125 resistance is still scarce: a set of 13 baseline reverse transcriptase mutations was previously identified in the DUET studies as having an effect on virological response to TMC125 [7-12]. Poveda et al. [12] suggested that efavirenz (EFV) might be less capable of inducing TMC125 resistance than nevirapine (NVP). Moreover, a longer duration of initial NNRTI treatment has been associated with increased evidence of in vitro TMC125 resistance [13] and the inclusion of NVP within the initial highly active antiretroviral therapy (HAART) regimen could result in a higher risk of virological failure and drug resistance compared with EFV [14]. This could limit the future use of TMC125 [15].

Recently, Tambuyzer et al. examined two TMC125 weighted genotypic scores (WGSs) [TBT (Tibotec, Mechelen, Belgium) and MGR (Monogram, San Francisco, CA, USA)], which produced similar results in defining susceptibility to TMC125 in treatment-experienced patients and were able to predict nonresponse to TMC125 in $\sim 60 \%$ of subjects enrolled in the DUET trials [16]. Nevertheless, there is a difference between mutations associated with TMC125 use (L100I, E138G, V179F/I, Y181C/I and H221Y), i.e. mutations that emerge with use of TMC125, and mutations associated with an altered response to TMC125 (V90I, A98G， L100I， K101E/H/P，V106I， E138A， V179D/T/F, Y181C/I/V and G190A/S).

To evaluate whether etravirine might be effective in patients failing therapy with current NNRTIs, we analysed the prevalence of etravirine mutations and possible determinants of genotypic resistance to this drug among sequences reported to a large Italian database.

\section{Materials and methods}

We retrospectively considered HIV-1 reverse transcriptase sequences obtained from the Italian Antiretroviral Resistance Cohort Analysis (ARCA; available at www.hivarca.net) database for a total of 2955 patients experiencing therapy failure with an NNRTI-based regimen at the time of blood drawing, and with complete treatment history available. These subjects had been selected on the basis of having a resistance test while failing their antiretroviral regimen (viral load $>1000$ HIV-1 RNA copies/mL). Patients were TMC125-naïve. Inclusion criteria were NNRTI-based regimen for at least 3 months, and an HIV RNA measurement and CD4 cell count available within 1 month.
Drug resistance mutations were interpreted following the latest International AIDS Society-USA (IAS-USA) panel list of mutations proposed to be TMC125-specific (www.iasusa. org; update in December 2008) [17]: V90I, A98G, L100I, K101E, K101P, K101H, V106I, E138A, V179D, V179F, V179T, Y181C, Y181I, Y181V, G190A, G190S and M230L. Given the high prevalence of mutation K103N among isolates resistant to other NNRTIs, we also added this mutation to our analysis.

The risk of developing at least three TMC125 resistanceassociated mutations (RAMs) [7,8] was assessed by means of binary logistic regressions models. Univariate and multivariate analyses were performed to estimate crude and adjusted relative risks (odds ratios, 95\% confidence intervals and Wald statistic) for gender, age, HIV RNA, CD4 cell count; and NVP, EFV, protease inhibitor (PI) and enfuvirtide (T20) exposure. Moreover, we considered the number of NNRTIs received and the duration of NNRTI therapy. The level of statistical significance was set at $P=0.05$. SPSS 15 for Windows was the statistical software package used for the analyses (SPSS, Chicago, IL, USA). Moreover, we conducted our analysis with the endpoint of having a TBT WGS $>2$, which has been reported to predict poor virological response to TMC125 in treatmentexperienced patients [16].

\section{Results}

A total of 5011 sequences obtained from 2955 patients were evaluated. Of these, 1241 subjects (42.0\%) were exposed only to NVP, 1053 (35.6\%) only to EFV, and 613 (20.7\%) to both NVP and EFV.

Of these 2955 patients, 2153 (72.9\%) presented with at least one TMC125 RAM. Among the sequences in ARCA, $68 \%$ had at least one and $9.8 \%$ at least three TMC125 RAMs, whereas $31 \%$ showed a WGS $>2$.

Among the samples with at least one RAM for TMC125 ( $n=3407)$, the mutations most frequently represented were Y181C (27\%), G190A (22.8\%), K101E (11.7\%) and A98G (9.3\%). K103N was present in 53.9\% of sequences. V179F, Y181V and G190S were present in 0.3\%, 1.0\% and 4.9\% of sequences, respectively. When at least three TMC125related mutations were found $(n=495)$, the mutations most frequently represented were G190A (62\%), Y181C (57.6\%) and K101E (44\%). K103N was present in 44.8\% of sequences. V179F, Y181V and G190S were present in 1.4\%, $1.0 \%$ and 13.5\% of sequences, respectively (Fig. 1). Among the samples with TBT WGS $>2 \quad(n=1553)$, the most frequent mutations were Y181C (59.3\%), G190A (26.7\%) and K101E (17.8\%); K103N was found in $40.1 \%$ of sequences. 
We also analysed the association between TMC125 RAMs and exposure to NVP and EFV: these mutations appeared more frequently in NVP- than EFV-treated patients: $90.2 \%$ of sequences from patients exposed to NVP vs. 35.2\% of sequences from patients exposed to EFV had mutation Y181C, and these percentages were, respectively, 84.7\% vs. 43.1\% for G190A, 72.7\% vs. 49.8\% for K101E, 100\% vs. 23.5\% for $\mathrm{Y} 181 \mathrm{~V}$, and $66.7 \%$ vs. 33.3\% for V179F. G190S appeared more frequently with exposure to EFV (81.4\% of sequences) than NVP (43.3\% of sequences).

Multivariate analysis revealed that male gender and being EFV- or NVP-experienced were associated with statistically significant increases in the risk of developing

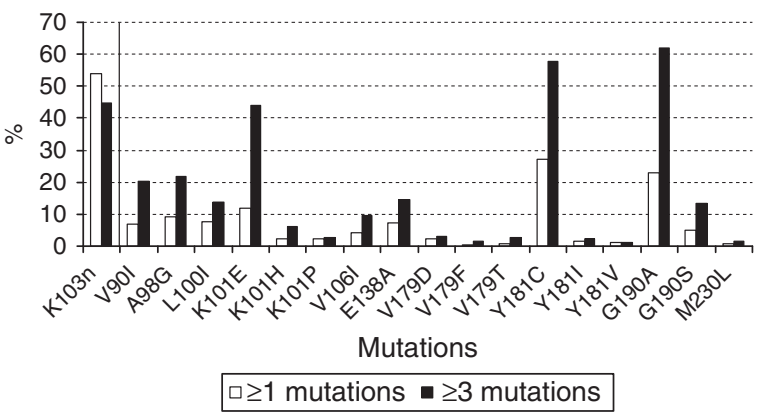

Fig. 1 Drug resistance mutations were interpreted following the latest International AIDS Society-USA (IAS-USA) panel list of mutations proposed to be etravirine (TMC125)-specific (www.iasusa. org; update in December 2008) [17]. There were 3407 sequences with at least one TMC125 resistance-associated mutation (RAM) and 495 sequences with at least three TMC125 RAMs. Mutation K103N was pictured as reference NNRTIs resistance mutation. three or more TMC125 RAMs. CD4 values $\geq 200$ cells $/ \mu \mathrm{L}$ and greater age (for each additional 10 years) were statistically protective factors, whereas PI and T20 experience and HIV RNA values did not show any statistically significant associations. Considering the duration of NNRTI treatment, every additional year of therapy represented a slightly increased risk of developing at least three TMC125 RAMs, although this was not statistically significant; also, being exposed to two NNRTIs, but not to one, produced an adjusted odds ratio (AOR) of 1.93 (95\% CI 0.74-5.07).

We then focused our attention on the risk of having a TBT WGS $>2$. As shown in Table 1, some differences were found in comparison to the analysis of at least three TMC125 RAMs. Of interest, a strong predictor of a decreased phenotypic susceptibility to TMC125 was a higher HIV RNA value (maximum risk at $>5 \log _{10}$ copies $/ \mathrm{mL}$ ), with the AOR increasing from 2.62 for HIV RNA ( $<3.7 \log _{10}$ copies/mL; 95\% CI 1.35-5.10; $\left.P=0.004\right)$ to 3.99 for HIV RNA ( $>5 \log _{10}$ copies/mL; 95\% CI 1.988.04; $P<0.001)$. NVP exposure retained an increased risk of a TBT WGS $>2$ (AOR 1.76; 95\% CI 1.42-2.18; $P<0.001$ ), whereas previous EFV treatment did not. Duration of NNRTI therapy and previous exposure to one NNRTI did not have any significant effect, whereas exposure to two NNRTIs still had a significant effect, with an AOR of 2.26 (95\% CI 1.05-4.88; $P=0.038)$.

\section{Conclusions}

The prevalence of TMC125-related mutations in the ARCA cohort was $68 \%$. According to the DUET studies $[7,8]$,

Table 1 Determinants of genotypic resistance [Tibotec (TBT) score $0-2$ or $>2$ ] to TMC125

\begin{tabular}{|c|c|c|c|c|c|c|}
\hline & Category & TBT score $0-2$ & TBT score $>2$ & AOR & $95 \% \mathrm{Cl}$ & $P$ \\
\hline Age (years) & & $42.8(42)$ & $41.4(40)$ & 0.80 & $(0.72-0.88)$ & $<0.001$ \\
\hline \multirow[t]{2}{*}{ Sex } & Female & $504(65.6)$ & $264(34.4)$ & 1 & & \\
\hline & Male & $1168(62.2)$ & $698(37.4)$ & 1.26 & $(1.05-1.51)$ & 0.015 \\
\hline \multirow[t]{4}{*}{ CD4 count } & $<200$ cells $/ \mu L^{*}$ & $298(56.4)$ & $230(43.6)$ & 1 & & \\
\hline & 200-399 cells $/ \mu \mathrm{L}$ & $408(66.7)$ & $204(33.3)$ & 0.70 & $(0.54-0.90)$ & 0.005 \\
\hline & $\geq 400$ cells $/ \mu \mathrm{L}$ & $450(71.4)$ & $180(28.6)$ & 0.57 & $(0.44-0.74)$ & $<0.001$ \\
\hline & ND & $516(59.7)$ & $348(40.3)$ & 0.99 & $(0.76-1.29)$ & 0.964 \\
\hline \multirow[t]{5}{*}{ HIV RNA } & $<0.7 \log _{10}$ copies $/ \mathrm{mL}^{*}$ & $69(86.3)$ & $11(13.8)$ & 1 & & \\
\hline & $<3.7 \log _{10}$ copies $/ \mathrm{mL}$ & $476(69.8)$ & $206(30.2)$ & 2.62 & $(1.35-5.10)$ & 0.004 \\
\hline & $3.7-5 \log _{10}$ copies $/ \mathrm{mL}$ & $585(60.5)$ & $382(39.5)$ & 3.67 & $(1.90-7.10)$ & $<0.001$ \\
\hline & $>5 \log _{10}$ copies $/ \mathrm{mL}$ & $138(54.5)$ & $115(45.5)$ & 3.99 & $(1.98-8.04)$ & $<0.001$ \\
\hline & ND & $404(62.2)$ & $248(38.0)$ & 2.92 & $(1.49-5.71)$ & 0.002 \\
\hline EFV-experienced & & $1008(66.7)$ & 504 (33.3) & 0.96 & $(0.78-1.17)$ & 0.665 \\
\hline NVP-experienced & & $974(58.5)$ & $690(41.5)$ & 1.76 & $(1.42-2.18)$ & $<0.001$ \\
\hline PI-experienced & & $1325(62.7)$ & 789 (37.3) & 1.10 & $(0.89-1.37)$ & 0.369 \\
\hline T20-experienced & & $54(66.7)$ & 27 (33.3) & 0.73 & $(0.45-1.19)$ & 0.207 \\
\hline
\end{tabular}

This multivariate analysis was conducted with the endpoint of having a TBT score $>2$.

Values in the TBT score columns are $n(\%)$, with the exception of mean (median) for age. AOR for age is for each additional 10 years.

${ }^{*}$ Reference category.

AOR, adjusted odds ratio; $\mathrm{Cl}$, confidence interval; ND, not determined; EFV, efavirenz; NVP, nevirapine; PI, protease inhibitor; T20, enfuvirtide. 
Y181C, G190A, K101E and A98G were the mutations more frequently represented. The DUET studies showed that at least three TMC125-associated mutations were required to impair the efficacy of the drug [7,8]. In our cohort, only $9.8 \%$ of sequences showed at least three TMC125associated mutations, suggesting that the existence of this condition is infrequent even in patients with evidence of resistance to the other NNRTIs. V179F, Y181V and G190S, which have the most pronounced effect on the response, were present in $<5 \%$ of sequences. When at least three TMC125 RAMs were present, the mutations most frequently represented were confirmed to be Y181C, G190A and K101E, but not A98G. In this setting, the prevalence of V179F, Y181C and G190S also increased.

Y181C, a common mutation which confers resistance to other NNRTIs and to TMC125 when associated with two or more TMC125 RAMs and which was highly prevalent $(32.2 \%)$ in the Tibotec data set [16], was associated with at least two mutations in a higher percentage of sequences in this study than found in the DUET studies (27\% vs. 15\%, respectively) [7], but it was present with V179F and G190S in $<5 \%$ of sequences. The association of Y181C with G190A, K101E and A98G was statistically significant. The prevalence of $\mathrm{V} 179 \mathrm{~F}$ was low, but when associated with at least two mutations was present in 57\% of sequences and was associated most frequently with Y181C, but was never associated with G190S or Y181V. Y181V and G190S, the other mutations with a large impact on response, were associated with at least two mutations in a low percentage of sequences.

The multivariate analysis of determinants of TBT WGS $>2$ demonstrated a protective effect of higher CD4 counts and a negative effect of higher HIV-RNA values, as expected. The use of EFV resulted in less NNRTI resistance than did the use of NVP. The pattern of resistance mutations suggests that subsequent virological suppression with TMC125-containing regimens may be more successful if previous treatments included EFV rather than NVP. The difference between exposure to NVP and EFV might be relevant in resource-limited settings where NVP is often used. The long-term use of NVP without optimized nucleoside reverse transcriptase inhibitor (NRTI) background therapy could lead to an accumulation of resistance mutations. This is of particular relevance in situations where second-line HAART regimens are difficult to obtain.

The use of both NNRTIs, rather than the duration of NNRTI exposure, had an impact on the occurrence of TBT WGS $>2$. As many HIV-positive patients still initiate therapy with an NNRTI, it is particularly important to take this evidence into consideration. Of note, lower CD4 counts $(<200$ cells $/ \mu \mathrm{L})$ and higher HIV RNA loads $\left(>3.7 \log _{10}\right.$ copies $/ \mathrm{mL}$ ) were related to a greater risk of a TBT score $>2$.
The judicious examination of subjects' therapeutic histories and the use of TBT WGS were found to be effective in predicting resistance to TMC125. The adoption of such tools is recommended for evaluating new antiretrovirals for clinical use.

\section{Acknowledgements}

The authors acknowledge the many patients and colleagues who have been a constant source of inspiration and Miss Valeria Vimercati for helping with the manuscript preparation.

Financial support. None.

\section{References}

1 Zaccarelli M, Tozzi V, Lorenzini P et al. Multiple drug class-wide resistance associated with poorer survival after treatment failure in a cohort of HIV-infected patients. AIDS 2005; 19: 1081-1089.

2 Perno C-F, Moyle G, Tsoukas C, Ratanasuwan W, Gatell J, Schechter M. Overcoming resistance to existing therapies in HIV-infected patients: the role of new antiretroviral drugs. J Med Virol 2008; 80: 565-576.

3 Andries K, Azijn H, Thielemans T et al. TMC125, a novel next-generation nonnucleoside reverse transcriptase inhibitor active against nonnucleoside reverse transcriptase inhibitor-resistant human immunodeficiency virus type 1 . Antimicrob Agents Chemother 2004; 48: 4680-4686.

4 Vingerhoets J, Azijn H, Fransen E et al. TMC125 displays a high genetic barrier to the development of resistance: evidence from in vitro selection experiments. $J$ Virol 2005; 79:

12773-12782.

5 Madruga JV, Cahn P, Grinsztejn B et al. Efficacy and safety of TMC125 (etravirine) in treatment-experienced HIV-1-infected patients in DUET-1: 24-week results from a randomised, double-blind, placebo-controlled trial. Lancet 2007; 370: 29-38.

6 Lazzarin A, Campbell T, Clotet B et al. Efficacy and safety of TMC125 (etravirine) in treatment-experienced HIV-1-infected patients in DUET-2: 24-week results from a randomised, double-blind, placebo-controlled trial. Lancet 2007; 370: 39-48.

7 Vingerhoets J, Buelens A, Peeters M et al. Impact of baseline NNRTI mutations on the virological response to TMC125 in the phase III clinical trials DUET-1 and DUET-2. Antivir Ther 2007; 12: S34.

8 Vingerhoets J, Clotet B, Peeters M et al. Impact of baseline NNRTI mutations on the virological response to TMC125 (etravirine, ETR) in the DUET-2 phase III clinical trials. 11th European AIDS Conference. Madrid, Spain, 24-27 October, 2007 [Abstract P7.3/05]. 
9 Tambuyzer L, Vingerhoets J, Azijn H et al. Development of a list of mutations associated with NNRTI resistance for use in clinical research. 5th European HIV Drug Resistance Workshop. Cascais, Portugal, 28-30 March, 2007 [Abstract 67].

10 Tambuyzer L, Vingerhoets J, Azijn H, Daems B, Picchio G, de Béthune MP. Emerging mutations in patients with virological failure on TMC125 in the DUET-1 and DUET-2 clinical trials. 6th European HIV Drug Resistance Workshop. Budapest, Hungary, 26-28 March, 2008 [Abstract 47].

11 Picchio G, Vingerhoets J, Staes M et al. Prevalence of TMC125 resistance-associated mutations in a large panel of clinical isolates. 15th Conference on Retroviruses and Opportunistic Infections. Boston, MA, 2-6 February, 2008 [Abstract 866].

12 Poveda E, Garrido C, de Mendoza C et al. Prevalence of etravirine (TMC-125) resistance mutations in HIV-infected patients with prior experience of non-nucleoside reverse transcriptase inhibitors. J Antimicrob Chemother 2007; 60: 1409-1410.

13 Llibre JM, Santos JR, Puig T et al. Prevalence of etravirineassociated mutations in clinical samples with resistance to nevirapine and efavirenz. J Antimicrob Chemother 2008; 62: 909-913.
14 van den Berg-Wolf M, Huppler Hullsiek K, Peng G et al. Virologic, immunologic, clinical, safety, and resistance outcomes from a long-term comparison of efavirenz-based versus nevirapine-based antiretroviral regimens as initial therapy in HIV-1-infected persons. HIV Clin Trials 2008; 9: 324-336.

15 MacArthur RD, Huppler Hullsiek K, Peng G et al. Failing therapy with efavirenz results in significantly fewer mutations limiting to etravirine than failing therapy with nevirapine: ontreatment analyses from the CPCRA FIRST Study. Antivir Ther 2008; 13 (Suppl 3): A141.

16 Tambuyzer L, Vingerhoets J, Azijn H, Hoogstoel A, Nijs S, Picchio G. Comparison of two etravirine weighted genotypic scores with phenotypic susceptibility and virological response data. 7th European HIV Drug Resistance Workshop, Stockholm, Sweden, 25-27 March, 2009 [Abstract 114].

17 Johnson V, Brun-Vézinet F, Clotet B et al. Update of the drug resistance mutations in HIV-1: December 2008. Top HIV Med 2008; 16: 138-145. 